2-1-1994

\title{
Resonances and Recurrences in the Absorption Spectrum of an Atom in an Electric Field
}

J. Gao

William \& Mary

John B. Delos

William \& Mary, jbdelos@wm.edu

Follow this and additional works at: https://scholarworks.wm.edu/aspubs

Part of the Physics Commons

\section{Recommended Citation}

Gao, J. and Delos, John B., Resonances and Recurrences in the Absorption Spectrum of an Atom in an Electric Field (1994). Physical Review A, 49(2), 869-880.

https://doi.org/10.1103/PhysRevA.49.869

This Article is brought to you for free and open access by the Arts and Sciences at W\&M ScholarWorks. It has been accepted for inclusion in Arts \& Sciences Articles by an authorized administrator of W\&M ScholarWorks. For more information, please contact scholarworks@wm.edu. 


\title{
Resonances and recurrences in the absorption spectrum of an atom in an electric field
}

\author{
J. Gao and J. B. Delos \\ Physics Department, College of William and Mary, Williamsburg, Virginia 23187 \\ and Institute for Theoretical Atomic and Molecular Physics, Harvard-Smithsonian Center for Astrophysics, \\ 60 Garden Street, Cambridge, Massachusetts 02138
}

(Received 28 January 1993; revised manuscript received 23 April 1993)

\begin{abstract}
We use closed-orbit theory to study the absorption spectrum of an atom in an electric field. In previous work we examined absorption spectra above the zero-field ionization threshold. Only one closed orbit exists there, and it is unstable. Now we examine the situation below threshold. Here, the orbit parallel to the electric field is stable and, as the energy decreases, many other closed orbits bifurcate out of it. These closed orbits have simple patterns, and the associated recurrences are most clear if the absorption spectrum is measured using a scaled-variables method. The relation between the semiclassical EinsteinBrillouin-Keller-Marcus (EBKM) theory and periodic-orbit or closed-orbit theory is examined: they are complementary methods in the same sense that energy and time are complementary variables in quantum mechanics. Our numerical calculations show that sinusoidal fluctuations contributed by the closed orbits combine into peaks, and these peaks are in the locations predicted by EBKM theory.

PACS number(s): 32.60. $+\mathrm{i}, 32.80 .-\mathrm{t}, 32.70 . \mathrm{Cs}, 05.45 .+\mathrm{b}$
\end{abstract}

\section{INTRODUCTION}

There are two complementary approaches to semiclassical theory: the Einstein-Brillouin-Keller-Marcus (EBKM) method of action quantization, which applies to integrable or near-integrable systems, and the periodic.orbit theory, which has primarily been applied to chaotic systems, but which can be used for integrable systems as well. The EBKM theory describes individual quantum states: it says that each discrete state is associated with an "eigentrajectory" or "eigentorus"-a regular quasiperiodic trajectory having appropriately quantized values of its action variables.

Periodic-orbit theory begins with a description not of individual states, but of recurrences. It asserts that each periodic orbit produces a sinusoidal fluctuation in the density of states as a function of energy. The closely related closed-orbit theory of atomic absorption spectra asserts that each electron orbit that is closed at the nucleus produces a sinusoidal fluctuation in the photoabsorption rate as a function of energy. In both cases, recurrences (returns of a particle to its original location in phase space or in configuration space) produce large-scale structures in quantum properties (sinusoidal fluctuations that are visible when we average over a number of individual states).

Whenever both theories are correct, they must agree with each other. The EBKM quantization rules must be expressible as a periodic-orbit sum and conversely, the sinusoidal fluctuations in the density of states must combine themselves into Dirac $\delta$ functions with unit weight at each eigenvalue. This relation was first shown by Berry and Tabor [1] in 1976.

In this paper we carry out a somewhat analogous study of closed-orbit theory for the absorption spectrum of an atom in an electric field [2]. For such a system, quantum mechanics tells us that there are no truly bound states, but only quasi-discrete resonances. However, the equations of motion are separable, a family of bound classical orbits exists, and these orbits constitute a two-parameter family of tori. EBKM quantization of these tori correctly predicts the energies of the resonances [3].

We will show that, also as a consequence of separability, the closed orbits of the electron have simple patterns. We will explain that associated recurrences will be most clear if the absorption spectrum is measured using a scaled-variables method, varying the photon energy and the applied electric field simultaneously. We will display numerically that the sinusoidal fluctuations indeed combine into peaks, and that these peaks are in the locations predicted by EBKM theory.

It follows that the EBKM theory and periodic-orbit or closed-orbit theory are complementary methods in the same sense that energy and time are complementary variables in quantum mechanics. EBKM theory predicts individual resonances on the energy axis, and the Fourier transform describes the recurrences. Closed-orbit theory predicts individual recurrences in time, and the Fourier transform give the resonances.

In this paper we also give some attention to a commonly overlooked aspect of periodic-orbit theory. The periodic-orbit sum contains denominators which vanish at certain points. Gutzwiller [4] noted the existence of these points, and he repaired the singularities, more or less, by throwing them away. This was adequate for his purposes, but not for ours, because the singularities correspond to real physical effects. They are points of classical frequency resonance between the period of an orbit and the period of stable oscillation across an orbit. The vanishing denominators produce bifurcations in classical mechanics and focal points in quantum mechanics. (A correct quantum treatment would replace the singularity by large finite amplitudes. Such a treatment is being developed, but it is not available yet.) Therefore we keep 
the singularities in the formulas, but carefully avoid them in our computations. We will show that the effects of these focal points, bifurcations, and frequency resonances should be visible in experimental measurements.

\section{CLOSED CLASSICAL ORBITS OF AN ATOMIC ELECTRON IN AN ELECTRIC FIELD}

Treating the electron's motion according to classical mechanics, we seek those orbits which go out from the nucleus and later return to it. The Hamiltonian is (Fig. 1)

$$
\begin{aligned}
& H=\frac{\mathrm{p}^{2}}{2 m}+\mathcal{V}(\rho, z)=E, \\
& \mathcal{V}(\rho, z)=\frac{-Z e^{2}}{\left(\rho^{2}+z^{2}\right)^{1 / 2}}+e F z .
\end{aligned}
$$

Transforming to scaled semiparabolic coordinates [5], and considering the case $L_{z}=0$, the Hamiltonian becomes

$$
\begin{aligned}
& h=\frac{1}{2}\left(p_{u}^{2}+p_{v}^{2}\right)+V(u, v)=2, \\
& V(u, v)=\frac{1}{2}\left(u^{4}-v^{4}\right)-\varepsilon\left(u^{2}+v^{2}\right), \\
& \varepsilon=E / F^{1 / 2} .
\end{aligned}
$$

The transformation from $(u, v)$ to $(\rho, z)$ is a $2 \rightarrow 1$ mapping, most easily described by polar coordinates in each space. Let $s=\left(u^{2}+v^{2}\right)^{1 / 2}, \quad \Theta=\tan ^{-1}(v / u)$ and $r=\left(\rho^{2}+z^{2}\right)^{1 / 2}, \quad \theta=\tan ^{-1}(\rho / z)$. Then $s=(2 r)^{1 / 2} F^{1 / 4}$, $\Theta=\theta / 2$. The total energy of the electron $E$ in (2.1), divided by the (electric field strength) ${ }^{1 / 2}$, plays the role of a force constant in (2.2). We consider the case $\varepsilon<0$. The transformed Hamiltonian (2.2) also has an effective po-

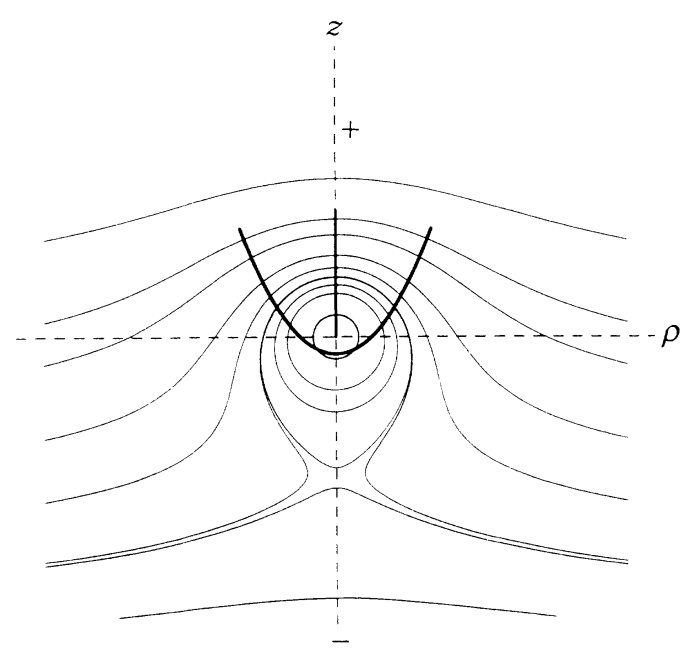

FIG. 1. Contour plot of the actual potential energy $\mathcal{V}(\rho, z)$. Around the origin is an infinitely deep Coulomb hole, and out the positive $z$ axis $\mathcal{V}(\rho, z)$ is increasing (approximately linearly at large $z$ ). For large negative $z, \mathcal{V}(\rho, z)$ decreases linearly, so there is a saddle point dividing the Coulomb hole from the escape region. A stable PO called the "parallel" orbit lies on the positive $z$ axis, and an unstable PO lies on a parabolic curve between the Coulomb hole and the saddle point. All other PO's organize themselves around these two fundamental ones. tential energy $V(u, v)$, which is shown in Fig. 2. In the $(u, v)$ plane it has two reflection symmetries,

$$
V(-u, v)=V(u,-v)=V(u, v) .
$$

A number of general results about such systems were established in Ref. [8]. In particular, any orbit which returns to the origin at time $T_{c}$ is periodic with period $2 T_{c}$. Therefore we can learn everything about the closed orbits by learning about the periodic orbits.

We define a Poincaré map $\left(v_{0}, p_{v_{0}}\right) \rightarrow\left(v_{1}, p_{v_{1}}\right)$, by integrating the $(u(t), v(t))$ equations, stopping whenever $u=0$ and $p_{u}>0$ [6]. Every periodic orbit (PO) of the differential equation is a periodic orbit of the map with the same period.

The associated surface of section (SOS) is shown in Fig. 3 at an energy below the zero-field ionization threshold. Since the Hamiltonian in Eq. (2.2) is separable, each curve is defined by the formula

$$
\begin{aligned}
& h_{v}\left(p_{v}, v\right)=\frac{1}{2} p_{v}^{2}+V_{v}(v)=e_{v}=\text { const }, \\
& V_{v}(v)=-\varepsilon v^{2}-\frac{1}{2} v^{4} .
\end{aligned}
$$

Because of the form of (2.4a), we call $e_{v}$ "the energy associated with $v$ motion" and $V_{v}(v)$ "the potential energy associated with $v$ motion" (though these quantities have no simple relationship to the energies defined in $\rho z$ coordinates). The SOS has two $X$ points and their associated separatrices, which define the boundary between orbits which are bound to the atom forever and those which escape. The separatrix trajectory can be obtained using Eqs. (2.4) and the separatrix condition: the energy $e_{v}$ equals the maximum of the potential energy associated

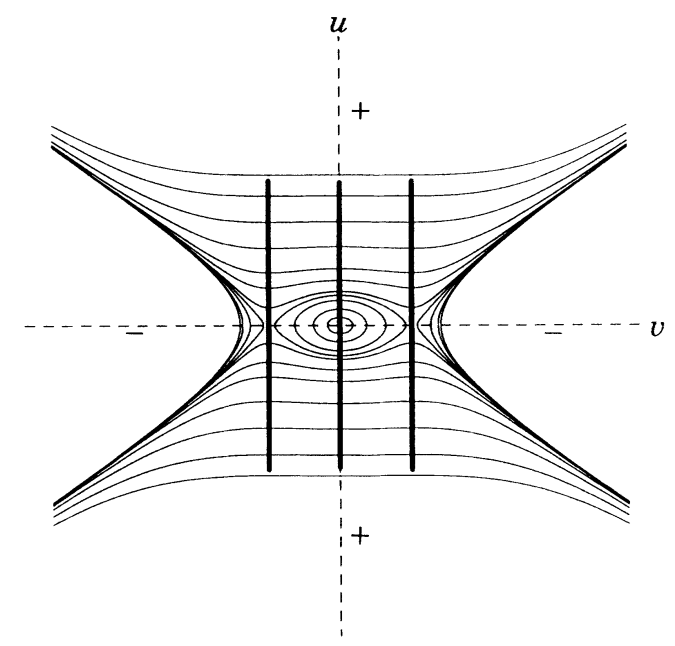

FIG. 2. Contour plot of the effective potential energy $V(u, v)$. The infinitely deep Coulomb hole has been converted into a quadratic well. Because of the 2:1 mapping from $(u, v)$ to $(\rho, z)$, the effective potential energy at large distances has acquired a quadrupolar structure, positive on the $\pm u$ axis and negative on the $\pm v$ axis. The stable PO ( the "parallel orbit") lies on the $u$ axis. The unstable PO is now two PO's that lie parallel to the $u$ axis and pass through saddle points of $V(u, v)$. 


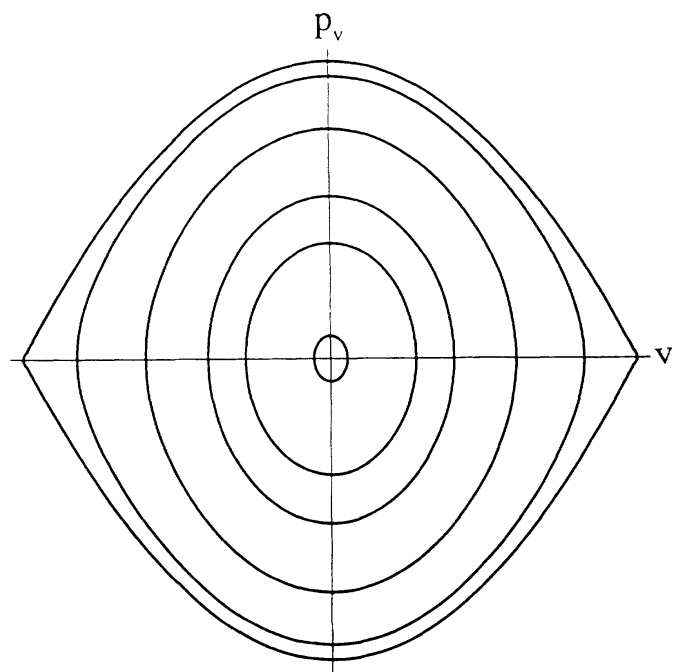

FIG. 3. Poincaré surface of section $\left(p_{v}, v\right)$ at $\varepsilon=-0.258$. The center $O$ point is the parallel orbit on the $u$ axis, and the $X$ points are the unstable orbits at the saddles of $V(u, v)$.

with $v$ motion,

$$
e_{v}=\max \left[V_{v}(v)\right]=\varepsilon^{2} / 2 .
$$

Thus the momentum $p_{v}$ on the separatrix equals

$$
p_{v}(v)=p_{s x}(v)= \pm\left(v^{2}+\varepsilon\right) .
$$

The plus and minus signs give the upper and lower branches of the separatrix, respectively. The $X$ points represent the unstable PO's which pass through the saddles in Fig. 2.

The stable PO at the center is the "parallel" orbit $P_{\|}$, which lies on the $u$ axis (the positive $z$ axis). The neighbors of this PO wind around it on the SOS. We seek all the other PO's that begin at the nucleus $(u=0, v=0)$, so they all start on the $p_{v}$ axis. In general, a period of $v$ motion is longer than a period of $u$ motion, and points on the SOS map clockwise; the angular coordinate of each point increases in each iteration of the map at an average rate $\bar{\alpha}\left(\varepsilon, p_{v_{0}}\right)$, which depends upon the scaled energy and the initial value of $p_{v}$. The winding rate on the SOS is the ratio of periods of $u$ to $v$ motion,

$$
\frac{\bar{\alpha}\left(\varepsilon, p_{v_{0}}\right)}{2 \pi}=\frac{T_{u}\left(\varepsilon, p_{v_{0}}\right)}{T_{v}\left(\varepsilon, p_{v_{0}}\right)} .
$$

In Fig. 4 each curve represents an iterate of the positive $p_{v}$ axis. At the energy displayed here, points close to the central point advance clockwise slightly more than $\frac{2}{5}$ of a revolution at each iteration of the map. Orbits more distant from the center wind more slowly, and especially they slow down in the vicinity of the $X$ points. Accordingly, successive iterates of the positive $p_{v}$ axis become ever more tightly wound spirals.

The general orbit is quasiperiodic. Periodic orbits occur whenever the average winding rate $\bar{\alpha}\left(\varepsilon, p_{v_{0}}\right) / 2 \pi$ is a rational fraction $m / l$. Every periodic orbit is a member of a continuous one-parameter family of periodic orbits, and these one-parameter families have shapes like Lissa-

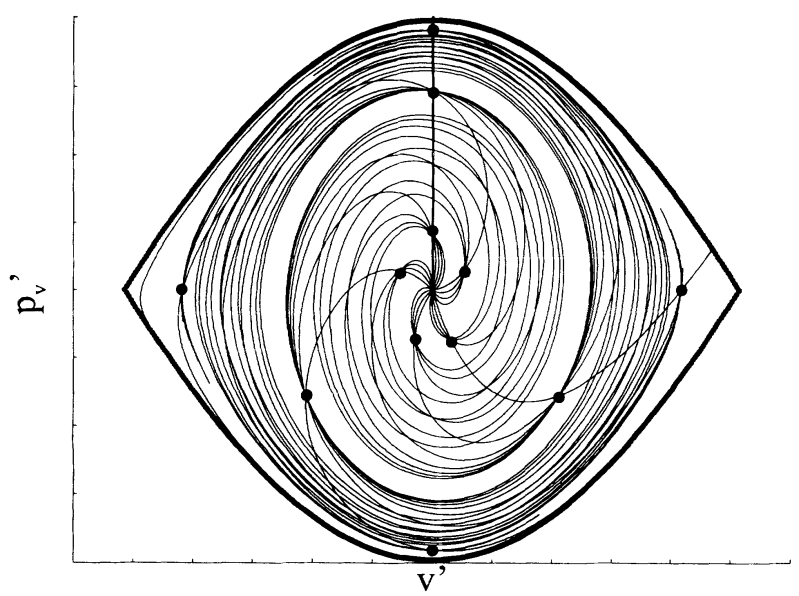

FIG. 4. The first 20 iterates of the positive $p_{v}$ axis at $\varepsilon=-0.258$. To display the structure near the separatrices, the coordinates have been stretched according to the following definitions: $\quad p_{v}^{\prime}=p_{v}\left(1+8 \sqrt{p_{v}^{2}+v^{2}}\right), \quad v^{\prime}=v\left(1+8 \sqrt{p_{v}^{2}+v^{2}}\right)$. Points close to the origin wind more rapidly than those further away. The first iterate is the curve that goes out from the origin at about " 5 o'clock" and curves over to the upper right separatrix. The second iterate goes out near "10 o'clock". Points where iterates intersect are PO's that touch the origin. The $2 / 5$, $1 / 3$, and $1 / 4$ orbits are marked by dots. On close examination one can find others, such as the $3 / 8$ orbit, which lies between $2 / 5$ and $1 / 3$.

jous figures (each family is distinguished by its frequency ratio, $m / l$, while within each family the members are distinguished by the initial relative phase of $u$ and $v$ motion).

Within each of these one-parameter families of PO's, there is one PO that touches the origin. These PO's show up in Fig. 4 as points where spirals intersect. Visible in Fig. 4 are orbits with average winding rate $2 / 5,3 / 8,1 / 3$, and $1 / 4$.

Since the only orbits that produce recurrences visible in the absorption spectrum are those that start at and later return to the origin, we see from Fig. 4 that the relevant closed orbits of each period are isolated, and that they fall into orderly patterns.

To understand the pattern let us think about the periods of $u$ and $v$ motion. At fixed energy $\varepsilon$, the period of $u$ motion is not very sensitive to $p_{v_{0}}$, and for our purposes we can say $T_{u}\left(\varepsilon, p_{v_{0}}\right) \approx T_{u}\left(\varepsilon, p_{v_{0}}=0\right)=T_{\|}$, the period of the parallel orbit. The period of $v$ motion is very sensitive to $p_{v_{0}}:$ this period is shortest when $p_{v_{0}}=0$, and it goes to infinity at the separatrix. Accordingly the average angular speed $\bar{\alpha}\left(\varepsilon, p_{v_{0}}\right)$ goes from a maximum at the parallel orbit

$$
\bar{\alpha}_{\max }(\varepsilon)=\bar{\alpha}(\varepsilon, 0)
$$

to zero at the separatrix. A PO occurs when $T_{u} / T_{v}$ is rational,

$$
m T_{v}\left(\varepsilon, p_{v_{0}}\right)=l T_{u}\left(\varepsilon, p_{v_{0}}\right)=T \approx l T_{\|} .
$$

Therefore, near each integer multiple $l$ of the period of 
the parallel orbit, there is a cluster of periodic orbits having

$$
T_{v}=\frac{l T_{u}}{m} \approx \frac{l T_{\|}}{m},
$$

where $m$ is any positive integer less than or equal to $m_{\max }(l, \varepsilon)$ with

$$
m_{\max }(l, \varepsilon)=\operatorname{int}\left[l \bar{\alpha}_{\max }(\varepsilon) / 2 \pi\right] .
$$

It is convenient to label these periodic orbits by the unreduced fraction $(\mathrm{m} / \mathrm{l})$. In this scheme the orbit labeled (for example) $6 / 15$ is the third repetition of the orbit labeled $2 / 5$. Then each primitive orbit closed at the origin is represented by a fraction expressed in lowest terms.

Including all repetitions of all these orbits, and including in addition all repetitions of the parallel orbit, near $m T_{\|}$there is a total of $m_{\max }(l, \varepsilon)+1$ periodic orbits. Conversely, there are exactly $m+1$ recurrences for each integer $l$ between

$$
\frac{2 \pi m}{\alpha_{\max }(\varepsilon)} \leq l<\frac{2 \pi(m+1)}{\alpha_{\max }(\varepsilon)} .
$$

This pattern is illustrated in Fig. 5, for the case $\varepsilon$ $=-0.266, \alpha_{\max } / 2 \pi \approx 0.41$. For this figure, we choose units of time such that the parallel orbit has "period 1." At $2 T_{\|}$, only the repetition of the parallel orbit is present. At $l=3$ a new sequence of periodic orbits appears having $m=1$, and $T_{u} / T_{v}=1 / l(l \geq 3)$. At $l=5$ another new sequence appears with $m=2$ and $T_{u} / T_{v}=2 / l(l \geq 5)$. For $l=6$, this sequence includes the $2 / 6$ orbit which is the repetition of the $1 / 3$ orbit. The next sequences start at $l=8,10,13, \ldots$.

Since each orbit that returns to the origin is half of a PO, the same pattern applies to recurrences in the absorption spectrum: at each value of $\varepsilon$, the recurrences can be labeled by every unreduced fraction $m / l$ less than or equal to $\alpha_{\max }(\varepsilon) / 2 \pi$. This winding rate can be calculated from the Jacobian matrix of the map

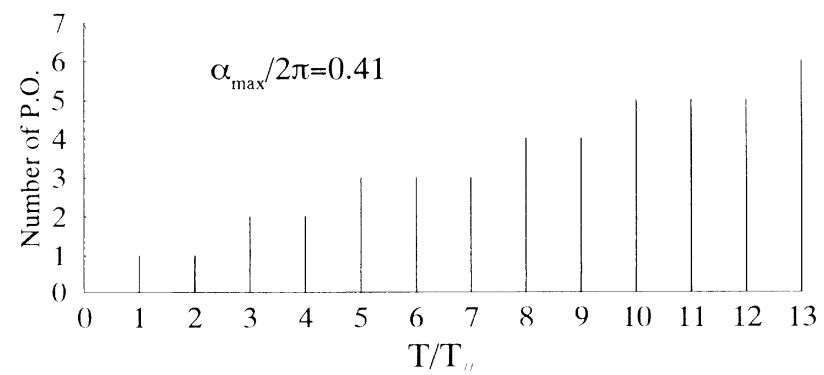

FIG. 5. Number of periodic orbits having period close to $l T_{\|}$ at $\varepsilon=-0.266$ where $\alpha_{\max } / 2 \pi=0.41$. There is one orbit with $T=T_{\|}$(the parallel orbit), one with $T=2 T_{\|}$(the first repetition of the parallel orbit), two near $T=3 T_{\|}$(the second repetition of the parallel orbit and the $1 / 3$ orbit), two near $T=4 T_{\|}$(the third repetition of the parallel orbit and the $1 / 4$ orbit), three near $T=5 T_{\|}$(the fifth repetition of the parallel orbit and the $1 / 5$ and $2 / 5$ orbit), and so on.

$$
\underline{J}(1)=\left[\begin{array}{ll}
\frac{\partial v_{1}}{\partial v_{0}} & \frac{\partial v_{1}}{\partial p_{v_{0}}} \\
\frac{\partial p_{v_{1}}}{\partial v_{0}} & \frac{\partial p_{v_{1}}}{\partial p_{v_{0}}}
\end{array}\right]
$$

evaluated at the $\mathrm{O}$ point (the parallel orbit):

$$
\begin{aligned}
& \operatorname{Tr} \underline{J}(1)=2 \cos \alpha_{\max }(\varepsilon), \\
& \pi<\alpha_{\max }<2 \pi \text { if } J_{12}<0, \\
& 0<\alpha_{\max }<\pi \text { if } J_{12}>0 .
\end{aligned}
$$

$\alpha_{\max }(\varepsilon)$ goes from $2 \pi$ as $\varepsilon \rightarrow-\infty$ to zero as $\varepsilon \rightarrow 0^{-}$. As $\alpha_{\max }(\varepsilon)$ decreases, each resonant PO moves to the $\mathrm{O}$ point and disappears. Conversely, as $\varepsilon$ decreases, and $\alpha_{\max }(\varepsilon)$ increases, new orbits bifurcate out of the parallel orbit. When $\alpha_{\max } / 2 \pi$ increases through the rational number $m / l$, a PO of period $l T_{\|}$is created, and it has $m$ cycles of $v$ motion in each period. We show a graph of $\alpha_{\max } / 2 \pi$ vs $\varepsilon$ in Fig. 6, and we also show some related bifurcation values in Table $\mathrm{I}$.

The geometrical structure of the orbits follows easily from the above considerations. For $m / l$ in lowest terms, there are $l$ full cycles of $u$ motion and $m$ full cycles of $v$ motion in a period of the orbit, or $l$ half cycles of $u$ motion and $m$ half cycles of $v$ motion before the first closure. Orbits labeled $1 / l$ climb the potential-energy barrier in a series of switchbacks, approaching the unstable PO on the ridge, then fall back again to the nucleus. Orbits labeled $2 / l$ do the same thing, but on falling back

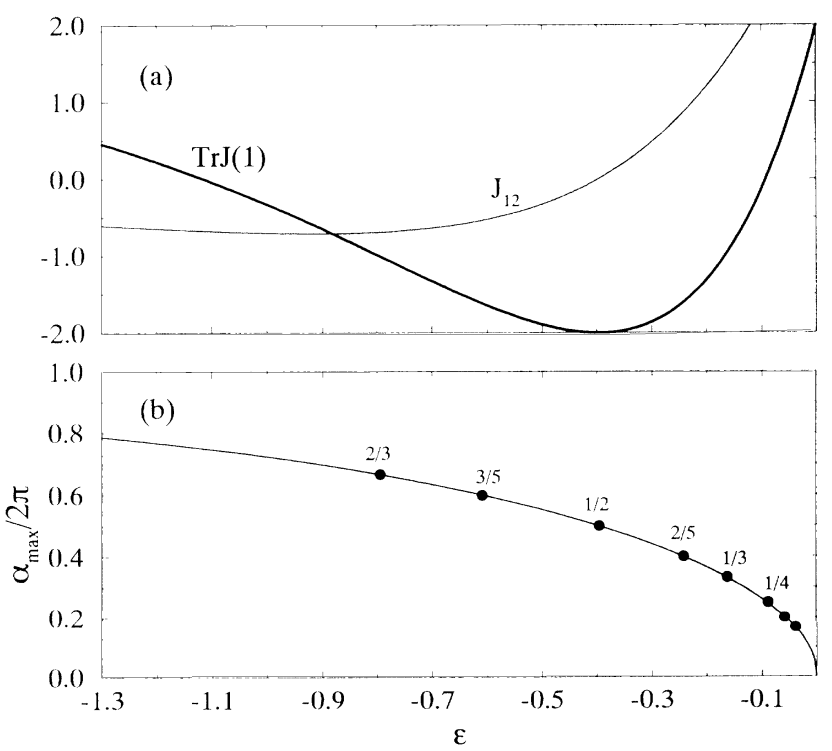

FIG. 6. (a) Trace of the Jacobi matrix of the Poincare map evaluated at the parallel orbit as a function of scaled energy. (b) Winding angle $\alpha_{\max } / 2 \pi$. As energy decreases, when $\alpha_{\max } / 2 \pi$ increases through the rational number $m / l$, the corresponding rational torus and its embedded closed orbit bifurcates out of the parallel orbit. Symbols indicate the bifurcation energies of the orbits $1 / 6,1 / 5,1 / 4,1 / 3,2 / 5,1 / 2,3 / 5$, and $2 / 3$. 
TABLE I. A list of the bifurcation values.

\begin{tabular}{clc}
\hline \hline$\alpha_{\max } / 2 \pi$ & \multicolumn{1}{c}{$\operatorname{Tr} \underline{J}(\varepsilon)$} & $\varepsilon$ \\
\hline $1 / 6$ & 1 & -0.0397 \\
$1 / 5$ & $-(\sqrt{5}+1) / 2$ & -0.0595 \\
$1 / 4$ & 0 & -0.0893 \\
$1 / 3$ & -1 & -0.164 \\
$2 / 5$ & $(\sqrt{5}-1) / 2$ & -0.243 \\
$1 / 2$ & -2 & -0.397 \\
$3 / 5$ & $-(\sqrt{5}+1) / 2$ & -0.610 \\
$2 / 3$ & -1 & -0.794 \\
\hline \hline
\end{tabular}

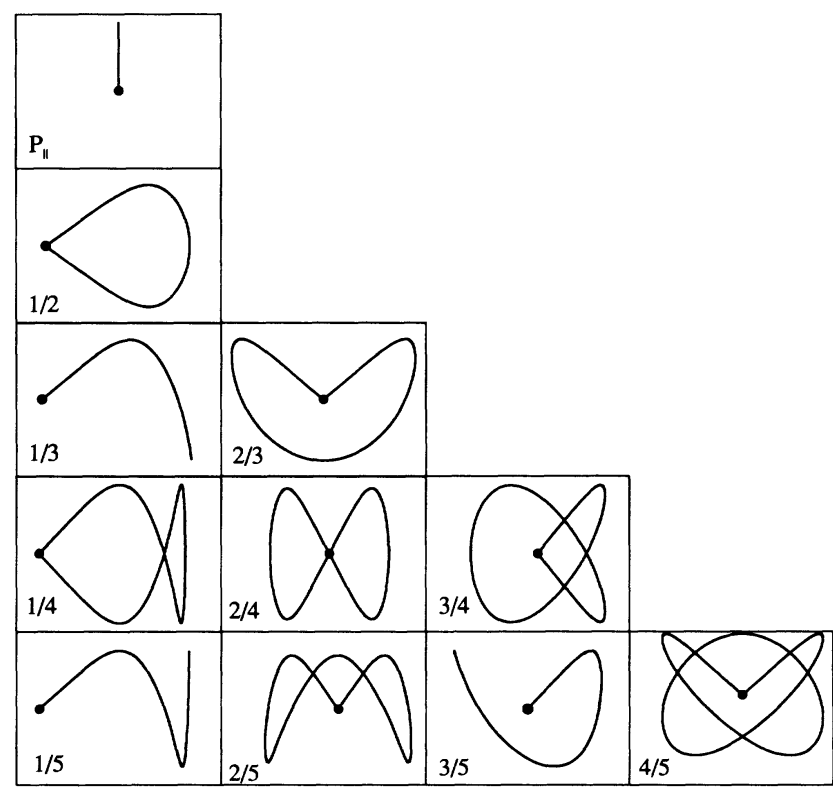

FIG. 7. Shapes of closed orbits in $(u, v)$ space.

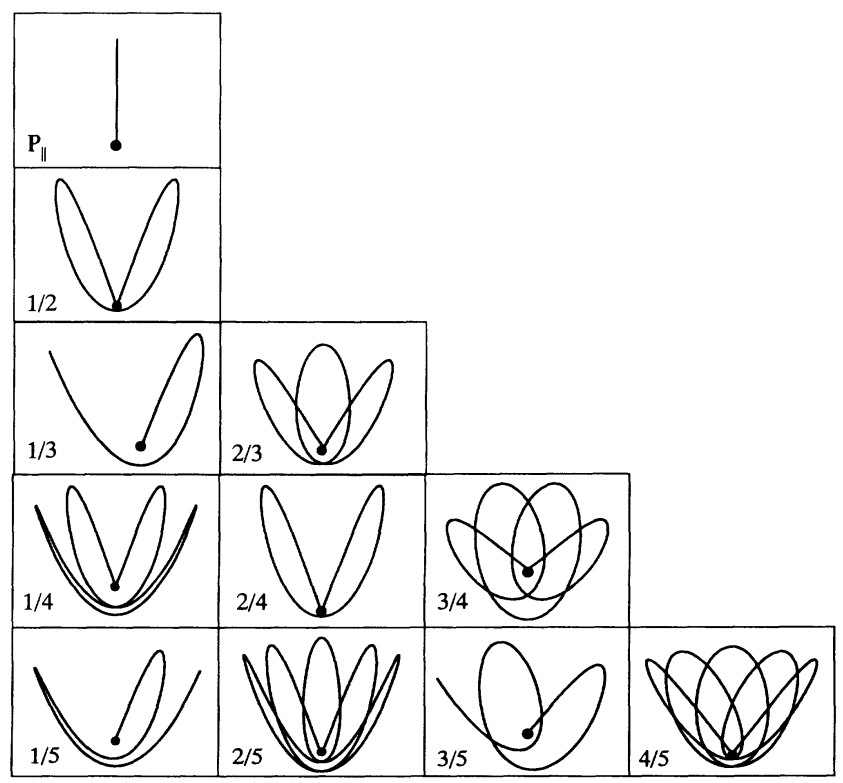

FIG. 8. Some closed orbits drawn in $(\rho, z)$ space. they miss the nucleus and climb the ridge on the other side before returning. Orbits labeled $m / l$ climb the two ridges alternately a total of $m$ times. Pictures of some orbits are shown in Figs. 7 and 8 [7].

\section{CLOSED-ORBIT THEORY OF PHOTOABSORPTION SPECTRA, AND SCALED-VARIABLE MEASUREMENT}

In Ref. [5] we derived formulas relating the absorption rate to properties of the closed orbits. Each closed orbit, (i.e., each recurrence) gives an oscillatory contribution to the absorption spectrum. The absorption spectrum can be defined quantitatively as a cross section for photon absorption, or as an oscillator-strength density $D f(E, F)$. The contribution of recurrences to the oscillator-strength density is called $D f_{1}(E, F)$.

The contribution of the parallel orbit to $D f_{1}(E, F)$ is

$$
\begin{aligned}
D f_{1}= & \left(E-E_{i}\right) 2^{9 / 2} \pi|Y(0) \widetilde{Y}(0)| F^{1 / 4} \\
& \times \sum_{n} \frac{\varepsilon^{1 / 2}}{\operatorname{Si}(\sqrt{2|\varepsilon| n \tau)}} \\
& \left.\quad \times \sin \mid n\left(S^{0} F^{-1 / 4}-\pi\right)-\frac{v_{n} \pi}{2}+\Delta_{0}\right),
\end{aligned}
$$

where $\mathscr{Y}(\theta)$ is the angular distribution of the outgoing wave, $\Delta_{0}=\arg [\widetilde{\mathcal{Y}}(0) \mathcal{Y}(0)]$ is a phase shift caused by the core, and $n$ is the label for repetitions of the closed orbit. The factor $\varepsilon^{1 / 2} / \mathrm{Si}(\sqrt{2|\varepsilon| n \tau})$ is proportional to the amplitude of the semiclassical returning wave, and $\tau$ is the scaled closure time of the orbit. $\operatorname{Si}(\sqrt{2|\varepsilon| \tau})$ means $|\sin (\sqrt{2|\varepsilon|} \mid \tau)|$ if $\varepsilon<0$ and $\sinh (\sqrt{2 \varepsilon} \tau)$ if $\varepsilon>0$. Finally, $S^{0}$ is the reduced action for the $0^{\circ}$ orbit, and $v_{n}$ is part of the Maslov index of the parallel orbit,

$$
v_{n}=\left\{\begin{array}{l}
0, \quad \varepsilon>0 \\
2 \text { int }\left(\frac{\sqrt{2|\varepsilon|} n \tau}{\pi}\right), \quad \varepsilon<0 .
\end{array}\right.
$$

For other closed orbits with $\theta_{i} \neq 0$, the formula for the contribution to the oscillator strength is

$$
\begin{gathered}
D f_{1}=\sum_{k} \frac{\left(E-E_{i}\right) 2^{11 / 2} \pi^{3 / 2}\left|\tilde{Y}\left(\theta_{f}^{k}\right) \mathcal{Y}\left(\theta_{i}^{k}\right)\right| F^{1 / 8} \sin \left(\theta_{i}^{k} / 2\right)}{\sqrt{J_{12}^{k}}} \\
\times \sum_{n} \frac{1}{\sqrt{n}} \sin \left\{n \mid S^{k} F^{-1 / 4}-\frac{\pi}{2} \mu^{k}-\frac{3}{2} \pi\right)+\frac{3 \pi}{4} \\
\left.+\Delta_{k}\right\} .
\end{gathered}
$$

Again $S^{k}$ is a reduced action for the $k$ th orbit, $\mu^{k}$ is its Maslov index, and $\Delta_{k}=\arg \left[\widetilde{\mathscr{Y}}\left(\theta_{f}^{k}\right) \mathscr{Y}\left(\theta_{i}^{k}\right)\right]$ with $\theta_{f}^{k}= \pm \theta_{i}^{k}$, $J_{12}^{k}$ is an element of the Jacobian matrix (2.12) evaluated at the first return of the $k$ th closed orbit. We called the entire coefficient of $\sin \{\}$ the "recurrence amplitude for the $n$th return of the $k$ th closed orbit." Its square is the corresponding "recurrence strength." The next three 
paragraphs give the derivation of Eq. (3.3), and the main argument begins again above Eq. (3.11).

To prove Eq. (3.3), we start from Eqs. (7.16) and (7.17) of Ref. [5],

$$
D f_{1}(E)=\sum_{m, k_{m}, n} \operatorname{Im}\left[C_{m, k_{m}, n} \exp \left(i \Delta_{m, k_{m}, n}\right)\right],
$$

where

$$
\begin{aligned}
C_{m, k_{m}, n} \exp \left(i \Delta_{m, k_{m}, n}\right)= & \left(E-E_{i}\right) 2^{19 / 4} \pi^{3 / 2} r_{b}^{-1 / 4} e^{2 i\left(8 r_{b}\right)^{1 / 2}} e^{-i 3 \pi / 4}\left(\sin \theta_{i}^{m, k_{m}} \sin \theta_{f}^{m, k_{m}}\right)^{1 / 2} \\
& \times A_{2}^{m, k_{m}, n} \exp \left[i\left(S^{m, k_{m}, n}-\frac{1}{2} \pi \mu^{m, k_{m}, n}\right)\right] \widetilde{\mathcal{Y}}_{m}\left(\theta_{f}^{m, k_{m}}\right) \mathcal{Y}_{m}\left(\theta_{i}^{m, k_{m}}\right)
\end{aligned}
$$

We now restrict ourselves to cases in which spin-orbit coupling is ignorable, and let $m=L_{z} / \hbar$ be equal to zero. Then the sum over $m$ in Eq. (3.4a) disappears, and the index $m$ can be dropped. In Eq. (3.4b) the action $S^{k, n}$ is defined to begin and end at a boundary radius $r_{b}$; the quantity $2\left(8 r_{b}\right)^{1 / 2}$ combines with $S^{k, n}$ to give the action all the way around the orbit, from origin to origin. The resulting action is written as $n S^{k}$.

The semiclassical amplitude $A_{2}^{k, n}$ can be simplified following the methods given in Ref. [8].

$$
A_{2}^{k, n}=\left[\sqrt{2 / r_{0}} \cos \left(\theta_{i} / 2\right) \cos \left(\theta_{f} / 2\right) J_{12}^{k}(n)\right]^{-1 / 2},
$$

where $J_{12}^{k}(n)=\partial v_{n} / \partial p_{v_{0}}$ evaluated at the $n$th closure (not the period) of the $k$ th closed orbit. Further simplifications follow because the system is separable. From Eq. (9.10) of Ref. [5] and letting $L_{z}=0$, we get

$$
\begin{aligned}
& p_{u}= \pm \sqrt{2 \cos \theta_{i}+2 \varepsilon u^{2}-u^{4}+2} \\
& p_{v}= \pm \sqrt{-2 \cos \theta_{i}+2 \varepsilon v^{2}+v^{4}+2}
\end{aligned}
$$

At the origin, $u_{i}=u_{f}=0, v_{i}=v_{f}=0$, so

$$
\begin{aligned}
& \left|p_{u_{i}}\right|=\left|p_{u_{f}}\right|=2 \cos \left(\theta_{i} / 2\right), \\
& \left|p_{v_{i}}\right|=\left|p_{v_{f}}\right|=2 \sin \left(\theta_{i} / 2\right) .
\end{aligned}
$$

Hence the closed orbits have the symmetry called "type 1 " in Ref. [8]. The element of the Jacobian matrix on the $n$th repetition is related to the element at the first closure by the formula

$$
\left|J_{12}^{k}(n)\right|=n\left|J_{12}^{k}(1)\right| \text {. }
$$

This follows from the fact that in this separable system, all periodic orbits (other than the stable parallel orbit and the unstable saddle-point orbit) have neutral stability (Appendix A).

Finally, the Maslov index for the $n$th repetition of a closed orbit is the sum of three terms: The first term is $n$ times the Maslov index for the first return. The second term is $2(n-1)$; every time the electron returns to the Coulomb center, there is a focusing effect that increases the Maslov index by 2 . The third term, $v_{n}$, counts any additional caustics that are not included in $n \mu_{1}$. Formulas for $v_{n}$ were given for stable and unstable orbits in Ref. [8]. Those formulas have a consistent limit for neutrally stable orbits; in our case we show in Appendix A that $J_{12} \operatorname{Tr} J<0$, so

$$
v_{n}=n-1 \text {. }
$$

It follows that

$$
\begin{aligned}
\mu_{n} & =n \mu_{1}+v_{n}+2(n-1) \\
& =n \mu_{1}+3(n-1) .
\end{aligned}
$$

Combining all these results, we obtain Eq. (3.3).

Now suppose that the absorption spectrum is measured at fixed scaled energy, varying the electric field $F$. The quantity that should be measured in the experiment is a reduced absorption rate,

$$
D f^{\prime}=\frac{D f_{1}}{E-E_{i}} F^{-3 / 16} \text {. }
$$

From Eqs. (3.1) and (3.3), the theoretical formula for this rate is

$$
D f^{\prime}=\sum_{k} \sum_{n} D_{k n} \sin \left(n S^{k} F^{-1 / 4}-\Phi^{k n}\right),
$$

where

$$
\begin{aligned}
& D_{0 n}=2^{9 / 2} \pi|\widetilde{\mathcal{Y}}(0) \mathcal{Y}(0)| F^{1 / 16} \frac{\varepsilon^{1 / 2}}{\operatorname{Si}(\sqrt{2|\varepsilon|} n \tau)}, \\
& \Phi^{0 n}=n \pi+v_{n} \frac{\pi}{2}-\Delta_{0}, \quad \theta_{i}=0
\end{aligned}
$$

and

$$
\begin{aligned}
& D_{k n}=\frac{2^{11 / 2} \pi^{3 / 2}\left|\mathcal{Y}\left(\theta_{i}^{k}\right) \tilde{\mathcal{Y}}\left(\theta_{i}^{k}\right)\right| F^{-1 / 16} \sin \left(\theta_{i}^{k} / 2\right)}{\sqrt{n J_{12}^{k}}}, \\
& \Phi^{k n}=n\left[\frac{\pi}{2} \mu^{k}+\frac{3}{2} \pi\right)-\frac{3 \pi}{4}-\Delta_{k}, \quad \theta_{i} \neq 0 .
\end{aligned}
$$

The "reduced recurrence amplitudes" $D_{k n}$ depend on the scaled energy and only weakly depend on the field strength. Over the range of field strength that might be used, $F^{1 / 16}$ and $F^{-1 / 16}$ do not change much, so an average field strength $F_{0}$ can be used instead, and therefore $D_{k n}$ is practically independent of $F$. At fixed scaled energy, the absorption spectrum would be regarded as a function of $w=F^{-1 / 4}$, and one would take its Fourier transform with respect to $w$.

[Let us note that the factor $F^{-3 / 16}$ in Eq. (3.11) is a compromise between $F^{-1 / 4}$ and $F^{-1 / 8}$. The recurrence amplitude of the $0^{\circ}$ orbit is proportional to $F^{1 / 4}$, while that for all other orbits is proportional to $F^{1 / 8}$. In our case, all recurrence amplitudes are comparable in magnitude, so we compromise. If one group or the other were to dominate, it would be better to use $F^{-1 / 4}$ or $F^{-1 / 8}$ in 
Eq. (3.11).]

In the preceding section, we explained that the orbits closed at the nucleus fall into orderly patterns such that each can be labeled by an unreduced fraction $m / l$. Let $S_{m / l}, T_{m / l}$, and $A_{m / l}$ be the action, return time, and semiclassical amplitude of the $m / l$ orbit. Since for large $l$ the orbits are approaching the unstable "ridge" orbit, it is not hard to show that in a sequence with $m$ fixed and $l$ increasing, the following relations hold at large $l$ :

$$
\begin{aligned}
& S_{m /(l+1)}-S_{m / l} \approx S_{\mathrm{un}} / 2, \\
& T_{m /(l+1)}-T_{m / l} \approx T_{\mathrm{un}} / 2, \\
& \frac{A_{m /(l+1)}}{A_{m / l}} \approx \exp \left(\frac{-\lambda_{\mathrm{un}}}{4 m}\right),
\end{aligned}
$$

where $S_{\text {un }}, T_{\text {un }}$, and $\lambda_{\text {un }}=2 \sqrt{|\varepsilon|} T_{\text {un }}$ are the action, and Liapunov factor of the unstable PO. A test of these approximations is given in Table II.

In the next two sections, we will use Eq. (3.12) to calculate the hydrogen absorption spectrum and its Fourier transform. The initial state of hydrogen is chosen to be $(2 s-2 p)$. The Y's are given in Eq. (5.12) of Ref. [5], and the $\Delta$ 's are equal to zero.

\section{RECURRENCE SPECTRA}

$D f^{\prime}$ in Eq. (3.12) is a sum of sinusoidal oscillations, each associated with a closed orbit or its repetitions. Hence its Fourier transform is a set of $\delta$ functions shown as the needles in Fig. 9. A needle is located at an action equal to the classical action of the associated closed orbit or its repetitions. The height of a needle is equal to the absolute square of the recurrence amplitude $D_{k n}$.

In experiments, the absorption rate would be measured and the Fourier transform would be calculated over a finite range. In our calculation, we use field-strength ranges that could be obtained in the laboratory (i.e.,

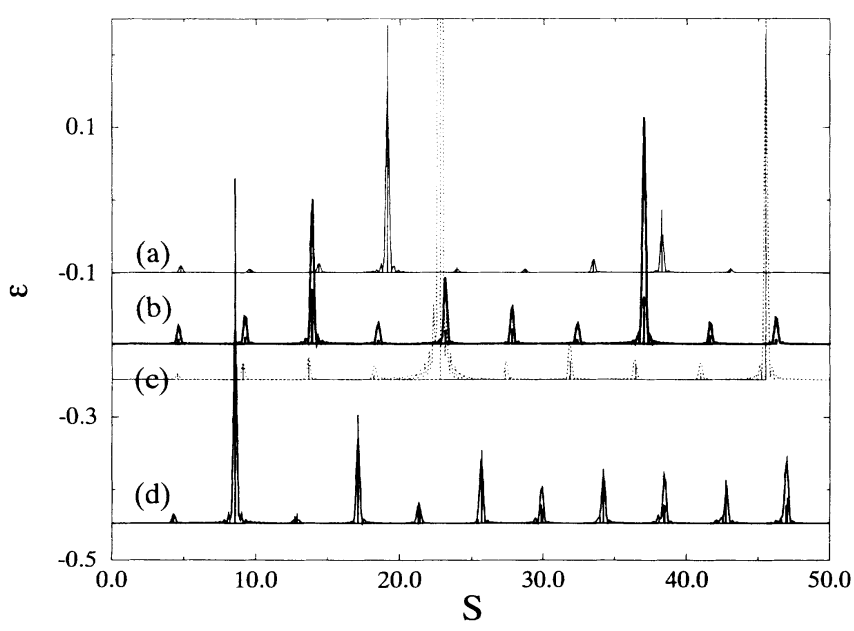

FIG. 9. Recurrence spectra (a) $\varepsilon=-0.0992$, near $1 / 4$ bifurcation; (b) $\varepsilon=-0.198$, near $1 / 3$ bifurcation; (c) $\varepsilon=-0.248$, near $2 / 5$ bifurcation; (d) $\varepsilon=-0.455$, near $1 / 2$ bifurcation. Each fixed $\varepsilon$ is a horizontal axis, and $|\widetilde{D} f(\mathscr{S})|^{2}$ is plotted on that axis.
$F=1000-3000 \mathrm{~V} / \mathrm{cm}$ and $F=8-10 \mathrm{~V} / \mathrm{cm})$, as the limits of the integral in the Fourier transformation. Let $w=F^{-1 / 4}$ and combine the labels $(k, n)$ into a single label $j$ for each recurrence. The Fourier transform

$$
\widetilde{D} f^{\prime}(\mathfrak{S})=\frac{2}{w_{2}-w_{1}} \int_{w_{1}}^{w_{2}} D f^{\prime} e^{-i \delta w} d w
$$

leads to a formula similar to that derived in Ref. [5],

$$
\begin{aligned}
\left|\widetilde{D} f^{\prime}(\mathcal{S})\right|^{2}= & \sum_{j} D_{j}^{2} \frac{\sin ^{2}\left(a x_{j}\right)}{\left(a x_{j}\right)^{2}} \\
+ & +2 \sum_{j} \sum_{l<j} D_{j} D_{l} \frac{\sin \left(a x_{j}\right) \sin \left(a x_{l}\right)}{\left(a x_{j}\right)\left(a x_{l}\right)} \\
& \quad \times \cos \left\{b\left(x_{j}-x_{l}\right)-\left(\Phi^{j}-\Phi^{l}\right)\right\},
\end{aligned}
$$

where

$$
a=\frac{w_{2}-w_{1}}{2}, \quad b=\frac{w_{2}+w_{1}}{2}, x_{j}=S_{j}-\mathcal{S},
$$

$S_{j}$ is given as $n S^{k}$ for the $n$th repetition of the $k$ th orbit. The $D_{j}$ 's are given by (3.12), with $F$ replaced by the average field for the assumed range of measurements. As a result of the finite range of measurements, each recurrence needle is spread out into a $(\sin x / x)^{2}$ peak, and overlapping peaks combine coherently. The power spectra are plotted for selected values of the scaled energy. We put several figures together in an overlay form as shown in Fig. 9.

Bifurcations are visible in the following way. Normally the recurrence amplitude of the parallel orbit is small, but near the $m / l$ bifurcation, the amplitude for the $l$ th return of the parallel orbit has a dramatic increase. For example, at $\varepsilon=-0.0992$, we see a large peak on the fourth return of the parallel orbit. This energy is close to that of the $1 / 4$ bifurcation in Table $I$. It is not surprising that a large peak also appears on the eighth return of the parallel orbit, for the parallel orbit is stable, and the newly created orbit $(1 / 4)$ is neutrally stable, so their repetitions should also have an effect on the spectrum. At $\varepsilon=-0.198$, there are large peaks at the third and fifth returns, and an especially large peak at the eighth return. Table I tells us that the $1 / 3$ bifurcation has just happened, and the $2 / 5$ bifurcation is about to happen. The large peak corresponds to the $3 / 8$ bifurcation, which happens at an energy higher than the $2 / 5$ bifurcation and very close to present energy. When $\varepsilon=-0.248$, we see a huge peak on the fifth return and its repetition, the tenth return. This energy is very close to the $2 / 5$ bifurcation, and the present semiclassical theory fails right on the bifurcation. The peaks on the fifth and tenth returns should be very large, but not infinite. We will discuss this in more detail in the next section.

If the absorption spectrum could be measured over a sufficiently large range of $w=F^{-1 / 4}$, bifurcations would also be visible in a second way. As $\varepsilon$ decreases, new recurrences are created, and, with sufficient resolution in $\mathcal{S}$, they appear as distinct peaks. In the present calculation the width of the peaks is too large for this to be visible. 


\section{RESONANCE SPECTRA}

\section{A. Complex EBKM theory}

In most spectroscopic experiments on atoms in electric fields, the field $F$ is fixed, and the photoabsorption rate is measured as a function of photon energy. In theoretical calculations, we would likewise fix $F$ and seek energy eigenvalues. However, in an electric field, atomic states are only quasibound, and they can be described by complex eigenvalues $E_{n}=E_{n}^{0}-i \Gamma_{n} / 2$. These values can be determined by seeking solutions to the Schrödinger equation subject to pure outgoing-wave boundary conditions [9].

Let us think for a moment about a complementary experiment. Suppose we hold the photon energy fixed and sweep the electric field. We would then want to know at what values $F_{n}$ does the photoabsorption spectrum have peaks, and what is the width of these peaks, $\Delta F_{n}$. For this purpose we might seek complex values of $F$ at which the Schrödinger equation (for fixed real $E$ ) has solutions which are purely outgoing [10].

In this paper, we are considering scaled-variable measurements: the scaled energy $\varepsilon=E / F^{1 / 2}$ is to be held fixed, and the parameter $w=F^{-1 / 4}$ is to be varied. The associated theory therefore involves a fixed real scaled energy and complex $w$ eigenvalues. We seek solutions to the Schrödinger equation in $(u, v)$ coordinates having fixed $\varepsilon$ and complex parameter $w$, and we locate eigenvalues $w_{n}=w_{n}^{0}-i \gamma_{n} / 2$ at those complex values of $w$ such that the $v$ factor in the wave function is purely outgoing. By analogy with usual quantum resonance theory, we claim that for real $\varepsilon$ and real $w$, the photoabsorption spectrum will show peaks in bands of width $\gamma_{n}$ around each $w_{n}^{0}$.

We implement this idea using a semiclassical approximation. The usual EBKM theory tells us that discrete quantum states correspond to "eigentrajectories," or tori on which the two action variables are quantized

$$
\begin{aligned}
& \oint p_{u} d u=\left(n_{u}+\frac{1}{2}\right) 2 \pi \hbar, \\
& \oint_{p_{v} d v}=\left(n_{v}+\frac{1}{2}\right) 2 \pi \hbar .
\end{aligned}
$$

These formulas apply to bound states.

In Appendix B, we derive appropriate quantization conditions for quasibound states in scaled variables. We show that the outgoing-wave condition is satisfied for values of $w_{n}$ obeying the two equations

$$
\begin{aligned}
& w \int_{0}^{a} \sqrt{2 e_{u}+2 \epsilon u^{2}-u^{4}} d u=\left(n_{u}+\frac{1}{2}\right) \pi, \quad n_{u}=0,1,2, \ldots \\
& \alpha+\frac{1}{4} i \beta \pi-\frac{1}{2}[\beta-\beta \ln (-\beta)]+\frac{1}{2} i \ln \left[\left(\frac{2}{\pi}\right)^{1 / 2} \Gamma\left[\frac{1}{2}+i \beta\right) \cosh (\pi \beta)\right]=\left(n_{v}+\frac{1}{2}\right) \pi, n_{v}=0,1,2, \ldots
\end{aligned}
$$

$\alpha$ and $\beta$ are associated with the action integrals of the well and barrier of $v$ motion,

$$
\alpha\left(w, e_{u}\right)=w \int_{0}^{c} \sqrt{-2 e_{u}+4+2 \varepsilon v^{2}+v^{4}} d v
$$

and

$$
\pi \beta\left(w, e_{u}\right)=-w \int_{c}^{e} \sqrt{2 e_{u}-4-2 \varepsilon v^{2}-v^{4}} d v .
$$

Using the method described in Appendix B, we used these quantization conditions to calculate the positions $w_{n}^{0}$ and widths $\gamma_{n}$ of quasibound resonances at two values of $\varepsilon$. The results are shown in Figs. 10(a) and 10(b) at $\varepsilon=-0.447$ and -0.179 . These scaled energies are close to the $1 / 2$ and $1 / 3$ bifurcations.

\section{B. The closed-orbit sum}

At the same scaled energies, we have calculated the absorption spectra using the closed-orbit formula (Sec. IV). We included all orbits with actions less than 16 times the action of the parallel orbit.

Several things are clear from these calculations.

(1) The closed-orbit sum gives peaks at the same values of $w$ that are found by the complex EBKM method.

(2) The closed-orbit sum gives predictions about the

\begin{tabular}{|c|c|c|c|c|}
\hline$l$ & $S_{m / l}$ & $\Delta S$ & $A_{m / l}$ & $f$ \\
\hline \multicolumn{5}{|c|}{$m=1$} \\
\hline \multirow[t]{2}{*}{3} & 13.968 & & 188.072 & \\
\hline & & 4.638 & & 3.826 \\
\hline \multirow[t]{2}{*}{4} & 18.606 & & 72.255 & \\
\hline & & 4.629 & & 3.305 \\
\hline \multirow[t]{2}{*}{5} & 23.235 & & 31.623 & \\
\hline & & 4.628 & & 3.144 \\
\hline \multirow[t]{2}{*}{6} & 27.863 & & 14.408 & \\
\hline & & 4.627 & & 3.094 \\
\hline \multirow[t]{2}{*}{7} & 32.490 & & 6.648 & \\
\hline & & $m=2$ & & \\
\hline \multirow[t]{2}{*}{7} & 32.578 & & 80.189 & \\
\hline & & 4.634 & & 3.606 \\
\hline \multirow[t]{2}{*}{8} & 37.212 & & 51.092 & \\
\hline & & 4.630 & & 3.371 \\
\hline \multirow[t]{2}{*}{9} & 41.842 & & 33.524 & \\
\hline & & 4.628 & & 3.240 \\
\hline \multirow[t]{2}{*}{10} & 46.470 & & 22.361 & \\
\hline & & 4.628 & & 3.165 \\
\hline \multirow[t]{2}{*}{11} & 51.098 & & 15.054 & \\
\hline & & $S_{\mathrm{un}} / 2=4.627$ & & $\lambda_{\mathrm{un}}=3.077$ \\
\hline
\end{tabular}
relative heights of peaks. The EBKM method can also give such information (as can other methods), but addi-
TABLE II. $\varepsilon=-0.179$, near $1 / 3$ bifurcation. $f \equiv-4 m \ln \left(A_{m /(l+1)} / A_{m / l}\right) . \quad S_{\text {un }}$ and $\lambda_{\text {un }}$ are the action and Liapunov factor for the unstable orbit. 
tional effort is required.

(3) As implemented here, the closed-orbit theory contains no information about tunneling. Therefore it should not give any reliable prediction of the widths of the peaks. The widths $\delta w$ of the structures shown in Figs. 10(a) and 10(b) are related to the largest-action orbits that are included in the sum, $S_{\max } \delta w \sim 2 \pi$. This raises the question, how can we incorporate tunneling into closed-orbit (or periodic-orbit) theory? Berry and Mount [11] suggested that each time a PO approaches the potential-energy barrier, there is a tunneling probability and a reflection probability. Their argument suggested that incorporating this reflection probability into the recurrence amplitude might give correct widths for the levels.

(4) Sharp truncation of the closed-orbit sum at $S_{\max }=16 S_{\|}$leaves spurious oscillations in the calculated absorption spectrum. We can get rid of these oscillations
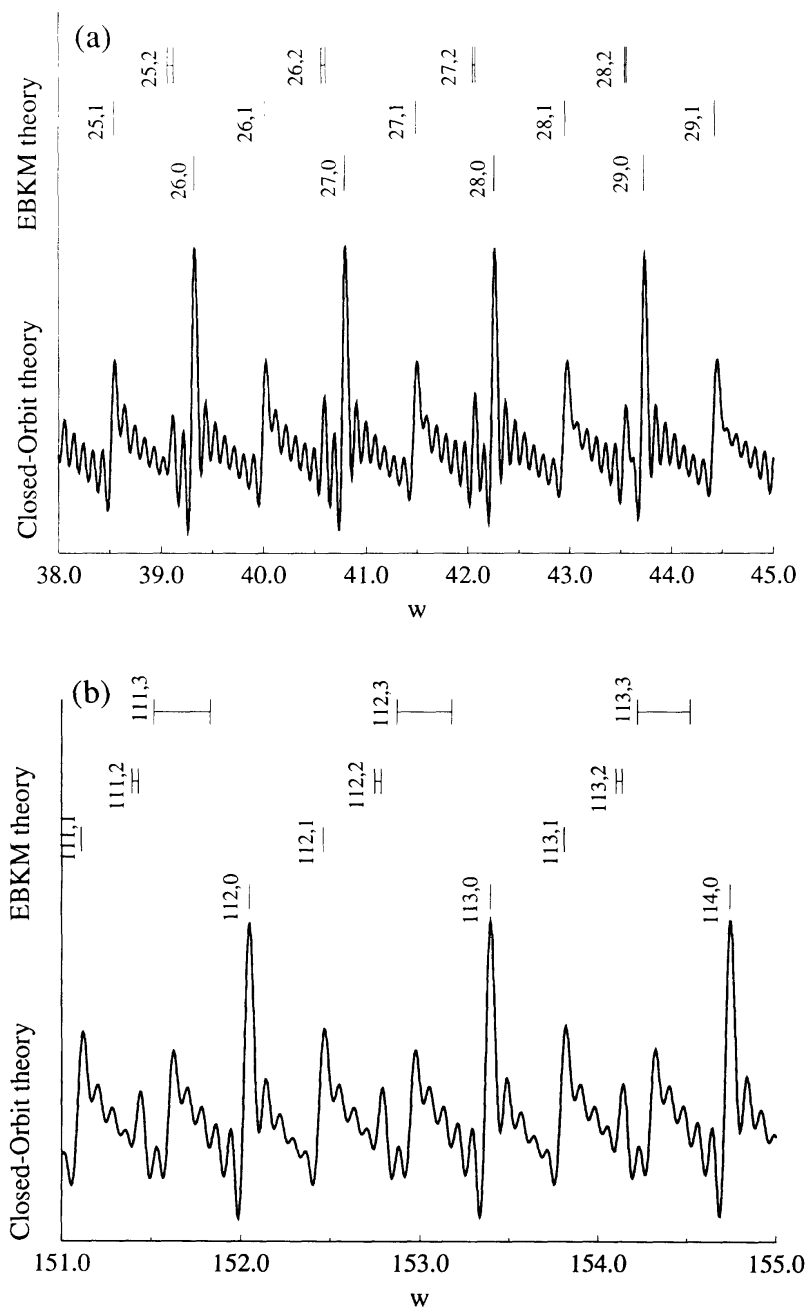

FIG. 10. Quantum resonance spectra-absorption vs $w=F^{-1 / 4}$ at fixed scaled energy $\varepsilon=E / F^{1 / 2}$. (a) $\varepsilon=-0.447$ near $1 / 2$ bifurcation. (b) $\varepsilon=-0.179$, near $1 / 3$ bifurcation. $w$ is dimensionless and is numerically equal to (electric field) ${ }^{-1 / 4}$ when the latter is measured in atomic units. In EBKM results, narrow resonances are indicated by lines labeled by quantum number $\left(n_{u}, n_{v}\right)$. Broad resonances are indicated by double lines. by using a gentle cutoff function for long orbits, but then the important peaks would be wider. Another interesting unsolved problem is can we approximately incorporate the effects of long orbits to eliminate spurious oscillations without loss of resolution? (One method was proposed in Ref. [12], but that method applies only to certain model systems having only unstable orbits.)

(5) This problem of long orbits is particularly challenging in the present implementation of the theory. The semiclassical denominator in Eq. (3.12b) has zeros or near-zeros at many values of $n$, leading to large highorder terms in the expansion, dubious convergence, and spurious short-wavelength oscillations.

We mentioned already that small denominators are produced by the same classical frequency resonances that produce bifurcations, and that at a bifurcation there is a focusing effect. This is illustrated in Fig. 11 at $\varepsilon=-0.397$, which corresponds to the $1 / 2$ bifurcation, where we show that family of trajectories close to the second return of the parallel orbit. The parallel orbit and its neighbors were integrated in $(u, v)$ space starting at $t=0$ and ending shortly after the second return of the parallel orbit. The family of returning orbits forms a shape associated with a cusp catastrophe, with the point of the cusp at the atom. At this value of $\varepsilon$, the semiclassical approximation predicts an infinite recurrence amplitude. There is a standard diffraction integral called a Pearcey function $[13,14]$ which gives the quantum wave function in the vicinity of a cusp. This integral must be modified so that it also incorporates the cylindrical symmetry of the present system. A full treatment will be given in a future paper.

(6) In view of the above remarks, the closed-orbit calculations describe one phenomenon with unexpected success. As $w$ decreases (and $F=w^{-4}$ increases), the resonances calculated by the EBKM method get wider, especially for $n_{v}=2,3$. Eventually their widths became com- (a) outgoing orbits

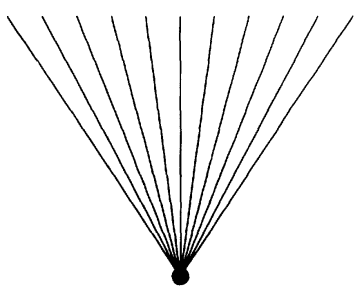

(b) returning orbits

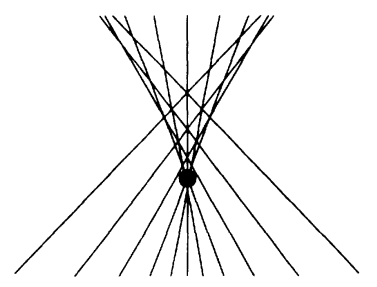

FIG. 11. Family of returning orbits near the second return of the parallel orbit at the $1 / 2$ bifurcation point, $\varepsilon=-0.447$. (a) Electrons go radially outward from the atom in every direction around the positive $u$ axis, which is directed upward. Later they return past the atom moving downward, and go out the negative $u$ axis. (b) On the second return, they are moving upward, and they form this cusp structure. Exactly at the bifurcation energy, the point of the cusp is at the nucleus, and the semiclassical formula for the recurrence strength diverges. For $\varepsilon$ above the bifurcation energy, the point of the cusp is above the atom, and only the parallel orbit touches the nucleus. For $\varepsilon$ below the bifurcation, the point of cusp is below the atom, and three lines pass through the nucleus. A new periodic orbit has been born. 
parable to the spacing between levels. At that point, one can stop thinking about them as quasidiscrete levels, and start thinking about them as part of the smooth background absorption. The corresponding peaks calculated by the present version of closed-orbit theory cannot get wider; however, their heights get smaller, and they do fade into the background.

Finally let us bring out the complementarity between resonance spectra and recurrence spectra. We explained that large peaks in recurrence spectra arise from classical frequency resonances between $u$ and $v$ motion. How do these same classical frequency resonances manifest themselves in the quantum resonance spectrum? We know that frequencies in classical mechanics correspond to energy gaps in quantum mechanics. The $1 / 2$ and $1 / 3$ classical resonances are visible in Fig. 10 in the sense that spacing between successive $n_{v}$ levels is approximately $1 / 2$ [Fig. 10(a)] or $1 / 3$ [Fig. 10(b)] of the spacing between successive $n_{u}$ levels. Of course this means that in the recurrence spectrum, the peak at $2 T_{\|}$or $3 T_{\|}$must be especially large. The quantitative description of the heights of these peaks will come from the correct treatment of the wave function at the focal point associated with the bifurcation (Fig. 11).

\section{CONCLUSION}

As stated in the Introduction, recurrence spectroscopy is complementary to resonance spectroscopy in the same sense that energy and time are complementary variables in quantum mechanics. In principle, they carry the same information. In practice, resonance spectroscopy naturally emphasizes individual quantum states and absorption lines (the small-scale structure of the spectrum), while recurrence spectroscopy naturally emphasizes early recurrences, which describe the large-scale structure of the resonance spectrum. Recurrence spectroscopy also provides an alternative way of thinking about spectra, in terms of closed orbits, classical frequency resonances, bifurcations, and focusing effects. It has been especially useful for studying nonseparable systems having a high density of states.

\section{ACKNOWLEDGMENTS}

This work was supported by the Jeffress Foundation, the Office of Naval Research, and the National Science Foundation through grants to the College of William and Mary and to the Institute for Theoretical Atomic and Molecular Physics. We specially thank the Institute for its hospitality.

\section{APPENDIX A: STABILITY MATRIX}

Here we consider closed orbits other than the parallel orbit. First we prove that the Jacobi matrix of the map at the $n$th closure of such a closed orbit has the form

$$
\underline{J}(n)=\left[\begin{array}{ll}
\frac{\partial v_{n}}{\partial v_{0}} & \frac{\partial v_{n}}{\partial p_{v_{0}}} \\
\frac{\partial p_{v_{n}}}{\partial v_{0}} & \frac{\partial p_{v_{n}}}{\partial p_{v_{0}}}
\end{array}\right]=\left[\begin{array}{cc} 
\pm 1 & \mp a \\
0 & \pm 1
\end{array}\right]
$$

with $a>0$.

All orbits satisfy the conservation law (2.4), so at the beginning and at the $n$th return,

$$
\begin{aligned}
& \frac{1}{2} p_{v_{0}}^{2}-\varepsilon v_{0}^{2}-\frac{1}{2} v_{0}^{4}=e_{v}\left(p_{v_{0}}, v_{0}\right), \\
& \frac{1}{2} p_{v_{n}}^{2}-\varepsilon v_{n}^{2}-\frac{1}{2} v_{n}^{4}=e_{v}\left(p_{v_{0}}, v_{0}\right) .
\end{aligned}
$$

Differentiating the second of these with respect to either $p_{v_{0}}$ or $v_{0}$

$$
\begin{aligned}
p_{v_{n}} \frac{\partial p_{v_{n}}}{\partial p_{v_{0}}}-2 \varepsilon v_{n} \frac{\partial v_{n}}{\partial p_{v_{0}}}-2 v_{n}^{3} \frac{\partial v_{n}}{\partial p_{v_{0}}} & =\frac{\partial e_{v}}{\partial p_{v_{0}}} \\
& =p_{v_{0}}, \\
p_{v_{n}} \frac{\partial p_{v_{n}}}{\partial v_{0}}-2 \varepsilon v_{n} \frac{\partial v_{n}}{\partial v_{0}}-2 v_{n}^{3} \frac{\partial v_{n}}{\partial v_{0}} & =\frac{\partial e_{v}}{\partial v_{0}} \\
& =-2 \varepsilon v_{0}-2 v_{0}^{3} .
\end{aligned}
$$

For any orbit that closes at the origin, $v_{n}=v_{0}=0$, so from (A2), (A3a), and (A3b), respectively, we have

$$
p_{v_{n}}^{2}=p_{v_{0}}^{2}
$$

and for all such orbits having $p_{v_{0}} \neq 0$ (i.e., all but the parallel orbit), from (A3a) and (A3b) we have

$$
\begin{aligned}
& \frac{\partial p_{v_{n}}}{\partial p_{v_{0}}}= \pm 1 \\
& \frac{\partial p_{v_{n}}}{\partial v_{0}}=0 .
\end{aligned}
$$

The Jacobi matrix must have $\operatorname{det} \underline{J}(n)=1$, so $\partial v_{n} / \partial v_{0}$ must also be \pm 1 .

Moreover, since the winding rate decreases as $p_{v_{0}}$ increases,

$$
\operatorname{sgn}\left(\frac{\partial v_{n}}{\partial p_{v_{0}}}\right)=-\operatorname{sgn}\left(\frac{\partial p_{v_{n}}}{\partial p_{v_{0}}}\right) .
$$

This establishes (A1). Also it shows that

$$
J_{12} \operatorname{Tr} \underline{J}<0 .
$$

Under this condition, according to (2.4c) of Ref. [8], the additional contribution to the Maslov index $v_{n}$ is $(n-1)$. Equation $(2.4 \mathrm{~b})$ of that reference gives the same result.

Finally, from formula (A1), it is easy to prove (3.8) by induction.

\section{APPENDIX B: EBKM RESONANCE LEVELS}

As explained in Sec. V, holding the scaled energy $\varepsilon$ fixed, we seek values of $w=w_{n}^{0}-i \gamma_{n} / 2$ such that there exists a solution to the Schrödinger equation in which the $v$ equation has outgoing waves only.

\section{Quantization conditions}

In scaled coordinates, the Schrödinger equation for an atomic electron in the electric field is 


$$
-\frac{1}{2 w^{2}} \Psi^{\prime \prime}-\left(\frac{1}{r}-z\right) \Psi=\varepsilon \Psi
$$

where $w=F^{-1 / 4}$. On writing the Laplacian operator in parabolic coordinates $u=\sqrt{r+z}, \quad v=\sqrt{r-z}$, $\varphi=\arctan (y / x)$, and multiplying the equation by $\left(u^{2}+v^{2}\right)$, Eq. (C1) assumes the form

$$
\begin{aligned}
\frac{-1}{2 w^{2}}\left[\frac{1}{u}\right. & \frac{\partial}{\partial u}\left(u \frac{\partial \Psi}{\partial u}\right)+\frac{1}{v} \frac{\partial}{\partial v}\left[v \frac{\partial \Psi}{\partial v}\right] \\
+ & {\left.\left[\frac{1}{u^{2}}+\frac{1}{v^{2}}\right) \frac{\partial^{2} \Psi}{\partial^{2} \varphi}\right] } \\
& -2 \Psi+\frac{1}{2}\left(u^{4}-v^{4}\right) \Psi=\varepsilon\left(u^{2}+v^{2}\right) \Psi .
\end{aligned}
$$

The above differential equation is separable by the assumption

$$
\Psi=\Psi_{u}(u) \Psi_{v}(v) e^{i m_{l} \varphi} / \sqrt{u v} .
$$

Hence with $m_{l}=0$, the functions $\Psi_{u}$ and $\Psi_{v}$ must satisfy the differential equations

$$
\begin{aligned}
& -\frac{1}{2 w^{2}} \Psi_{u}^{\prime \prime}-\left(\frac{1}{8 w^{2} u^{2}}+\varepsilon u^{2}-\frac{1}{2} u^{4}\right) \Psi_{u}=e_{u} \Psi_{u}, \\
& -\frac{1}{2 w^{2}} \Psi_{v}^{\prime \prime}-\left(\frac{1}{8 w^{2} v^{2}}+\varepsilon v^{2}+\frac{1}{2} v^{4}\right) \Psi_{v}=e_{v} \Psi_{v},
\end{aligned}
$$

where $e_{v}+e_{u}=2$. Here the parameter $1 / w^{2}$ is analogous to $\hbar^{2}$ in the normal Schrödinger equation.

The singular terms $\left(-1 / 8 w^{2} u^{2}\right)$ and $\left(-1 / 8 w^{2} v^{2}\right)$ do not arise in the classical equation of motion. When we use a semiclassical approximation to construct the wave functions, we should not include those terms. The WKB approximation to the solution to (B4) and (B5) is inaccurate near $u=0$ or $v=0$. Following the idea of Langer $[15,16]$, we should change variables to $x=\ln u$, and then apply the WKB approximation to the resulting equation. The effect of this transformation is to "erase" the singular terms from semiclassical formulas.

When this is done, the action integral associated with $u$ motion has the form $\int \sqrt{2 e_{u}+2 \varepsilon u^{2}-u^{4}} d u$. To get the quantization condition we use the following argument. The coordinates $(u, v)$ are originally defined to be positive. A "cycle" of $u$ motion starts at $u=0$, goes to a turning point at positive $u$, and returns to $u=0$, where the momentum $p_{u}$ is reflected, so that $u$ remains positive. The line $u=0$ is the positive $z$ axis, and on this axis the wave function has a focus. We have shown elsewhere that in each return to $u=0$, the Maslov index increases by 1 . Accordingly, we should treat $u=0$ as an ordinary turning point. Then the $u$-quantization condition is

$$
\begin{array}{r}
\oint_{p_{u}} d u=2 \int_{0}^{a}\left[2 e_{u}+2 \varepsilon u^{2}-u^{4}\right] d u=\left(n_{u}+\frac{1}{2}\right) \frac{2 \pi}{w}, \\
n_{u}=0,1,2, \ldots .
\end{array}
$$

$a$ is the zero of the integrand.

The $v$ motion has a barrier. We apply the same arguments at $v=0$, but we apply an "outgoing-wave only" boundary condition at large $v$. Then the complex semiclassical quantization condition is $[17,18]$

$$
\alpha+\frac{1}{4} i \beta \pi-\frac{1}{2}[\beta-\beta \ln (-\beta)]+\frac{1}{2} i \ln \left[\left(\frac{2}{\pi}\right)^{1 / 2} \Gamma\left[\frac{1}{2}+i \beta\right] \cosh (\pi \beta)\right]=\left(n_{v}+\frac{1}{2}\right) \pi, \quad n_{v}=0,1,2, \ldots
$$

where $\alpha\left(w, e_{u}\right)$ is the action integral associated with the well,

$$
\alpha\left(w, e_{u}\right)=w \int_{0}^{c} \sqrt{4-2 e_{u}+2 \varepsilon v^{2}+v^{4}} d v,
$$

and $\pi \beta\left(w, e_{u}\right)$ is the one for the barrier,

$$
\pi \beta\left(w, e_{u}\right)=-w \int_{c}^{d} \sqrt{2 e_{u}-4-2 \varepsilon v^{2}-v^{4}} d v .
$$

In Eqs. (B8) and (B9) $c\left(e_{u}\right), d\left(e_{u}\right)$ are (in general complex) zeros of the integrands; when real they are the classical turning points.

\section{Calculations}

Our parameter $w$ is related to $e_{u}$ in a very simple manner through (B6),

$$
w=w\left(e_{u}\right)=\frac{\left(n_{u}+\frac{1}{2}\right) \pi}{\int_{0}^{a} \sqrt{2 e_{u}+2 \varepsilon u^{2}-u^{4}} d u} .
$$

On plugging this formula for $w\left(e_{u}\right)$ into (B8) and (B9), the integrals $\alpha\left(w\left(e_{u}\right), e_{u}\right)$ and $\beta\left(w\left(e_{u}\right), e_{u}\right)$ become functions of the single variable $e_{u}$. Using these functions, Eq. (B7) is a quantization condition to determine $e_{u}$. It can be written in the form $F\left(e_{u}\right)=0$, and it can be solved by a complex-plane extension of the Newton-Raphson method.

Once $e_{u}$ is determined by this method, we put it back into (B10) to obtain the complex eigenvalues $w_{n}$. Results are shown in Fig. 10.
[1] M. V. Berry and M. Tabor, Proc. R. Soc. London, Ser. A 349, 101 (1976).

[2] We mention here some related work of other groups. U. Eichmann, K. Richter, D. Wintgen, and W. Sandner,
Phys. Rev. Lett. 61, 2438 (1988); G. Alber, Phys. Rev. A 40, 1321 (1989); E. B. Bogomol'nyi, Pis'ma Zh. Eksp. Teor. Fiz. 47, 445 (1988) [JETP Lett. 47, 526 (1988)].

[3] D. Farrelly and W. P. Reinhardt, J. Phys. B 16, 2103 
(1983).

[4] M. C. Gutzwiller, J. Math. Phys. 12, 343 (1971).

[5] J. Gao and J. B. Delos, Phys. Rev. A 46, 1449 (1992); 46, 1455 (1992).

[6] In other work [8], we have often used a Poincaré "half map," in which we record $\left(v, p_{v}\right)$ whenever $u=0$ regardless of the sign of $p_{u}$. For the present paper, the half map has no advantages.

[7] Somewhat similar results have been derived in a more general way by R. C. Churchill, G. Pecelli, and D. L. Rod, Arch. Ration. Mech. Anal. 73, 313 (1980).

[8] J. M. Mao, J. Shaw, and J. B. Delos, J. Stat. Phys. 68, 51 (1992).

[9] L. D. Landau and E. M. Lifshitz, Quantum Mechanics, 3rd ed. (Pergamon, New York, 1977).

[10] In scattering theory, one can use a fixed real angular momentum and calculate complex energies (Siegert poles), or alternatively, one can use a fixed real energy and calculate complex angular momentum eigenvalues (Regge poles).

[11] M. V. Berry and K. E. Mount, Rep. Prog. Phys. 35, 315 (1972).

[12] R. Aurich and F. Steiner, Proc. R. Soc. London, Ser. A 437, 693 (1992).

[13] M. V. Berry and C. Upstill, Prog. Opt. 18, 257 (1980).

[14] J. N. L. Connor, Mol. Phys. 26, 1217 (1973); J. N. L. Connor and D. Farrelly, J. Chem. Phys. 75, 2831 (1981).

[15] J. B. Delos, S. K. Knudson, S. D. Sikora, R. L. Waterland, and S. Whitworth, Phys. Rev. A 37, 4582 (1988).

[16] R. E. Langer, Phys. Rev. 51, 669 (1937).

[17] J. N. L. Connor, Mol. Phys. 31, 1181 (1976); 25, 1496 (1973); 15, 621 (1968); 23, 717 (1972).

[18] J. B. Delos and C. E. Carlson, Phys. Rev. A 11, 210 (1975). 